\title{
BMJ Global Health Community health workers improve disease control and medication adherence among patients with diabetes and/or hypertension in Chiapas, Mexico: an observational stepped-wedge study
}

\author{
Patrick M Newman, ${ }^{1}$ Molly F Franke, ${ }^{2}$ Jafet Arrieta, ${ }^{3}$ Hector Carrasco, ${ }^{3}$ \\ Patrick Elliott, ${ }^{4}$ Hugo Flores, ${ }^{5}$ Alexandra Friedman, ${ }^{6}$ Sophia Graham, ${ }^{7}$ \\ Luis Martinez, ${ }^{8}$ Lindsay Palazuelos, ${ }^{9}$ Kevin Savage, ${ }^{2}$ Hayley Tymeson, ${ }^{10}$ \\ Daniel Palazuelos ${ }^{5}$
}

To cite: Newman PM, Franke MF, Arrieta J, et al. Community health workers improve disease control and medication adherence among patients with diabetes and/or hypertension in Chiapas, Mexico: an observational stepped-wedge study. BMJ Glob Health 2018;3:e000566. doi:10.1136/ bmjgh-2017-000566

Handling editor Valery Ridde

Received 11 September 2017 Revised 2 November 2017 Accepted 15 November 2017

\begin{abstract}
Background Non-communicable diseases (NCDs) contribute greatly to morbidity and mortality in lowincome and middle-income countries (LMICs). Community health workers (CHWs) may improve disease control and medication adherence among patients with NCDs in LMICs, but data are lacking. We assessed the impact of a CHWled intervention on disease control and adherence among patients with diabetes and/or hypertension in Chiapas, Mexico.

Methods We conducted a prospective observational study among adult patients with diabetes and/or hypertension, in the context of a stepped-wedge roll-out of a CHW-led intervention. We measured self-reported adherence to medications, blood pressure and haemoglobin A1c at baseline and every 3 months, timed just prior to expansion of the intervention to a new community. We conducted individual-level mixed effects analyses of study data, adjusting for time and clustering by patient and community. Findings We analysed 108 patients. The CHW-led intervention was associated with a twofold increase in the odds of disease control (OR 2.04, 95\% Cl 1.15 to 3.62). It was also associated with optimal adherence assessed by 30-day recall (OR 1.86; 95\% Cl 1.15 to 3.02) and a positive self-assessment of adherence behaviour (OR 2.29; $95 \% \mathrm{Cl}$ 1.26 to 4.15$)$, but not by 5 -day recall.

Interpretation A CHW-led adherence intervention was associated with disease control and adherence among adults with diabetes and/or hypertension. This study supports a role of CHWs in supplementing comprehensive primary care for patients with NCDs in LMICs.

Trial registration number NCT02549495.
\end{abstract}

\section{INTRODUCTION}

For numbered affiliations see end of article.

Correspondence to Dr Patrick M Newman; Patrick.Newman@ucsf.edu
WHO identifies the rising prevalence of non-communicable diseases (NCDs) such as type II diabetes mellitus (diabetes) and cardiovascular disease as a major challenge for social and economic development in the

\section{Key questions}

What is already known about this topic?

- Community health workers (CHWs) represent a potentially effective force in helping to address the rising burden of noncommunicable disease (NCDs) in low-income and middle-income countries (LMICs).

- There is a lack of data evaluating the effectiveness of CHWs in improving outcomes among patients with NCDs in LMICs.

What are the new findings?

- A CHW-delivered intervention focusing on disease management and medication adherence improved disease control and adherence to medications among patients with diabetes and hypertension in rural Mexico, when added to a functioning comprehensive primary care system.

- These data provide the first prospective evidence of the effectiveness of CHWs in improving disease outcomes among patients with NCDs in Latin America.

Recommendations for policy

- CHWs have a role in health systems strengthening to improve disease outcomes among patients with NCDs in LMICs.

- Health systems that already provide comprehensive clinic-based primary care for patients with NCDs can further improve adherence, and clinical outcomes, by incorporating CHWs.

21st century. ${ }^{2}$ Patients in low-income and middle-income countries (LMICs) bear a disproportionate burden of morbidity and mortality from NCDs, ${ }^{2}$ and within-LMICs, patients with lower socioeconomic status have worse NCD outcomes and a higher prevalence of NCD risk factors such as hypertension. ${ }^{3}$ 
In Mexico, diabetes and ischaemic heart disease are the two leading causes of disability-adjusted life years as of 2015, with a rising prevalence in almost all demographic groups. ${ }^{45}$ Studies using national survey data estimate the proportion of patients with diabetes meeting definitions of clinical control at $8 \%,{ }^{6}$ and $57 \%$ among patients with hypertension. ${ }^{7}$ Effective coverage for hypertension, a WHO benchmarking tool used by Mexico defined as the fraction of potential health gain that can be delivered by the health system that is actually delivered, is estimated to be $23 \% .{ }^{8}$ Addressing NCDs and NCD risk factors is a priority for the Mexican Ministry of Health. ${ }^{45}$

Community health workers (CHWs) play an important and growing role in health systems worldwide, and there is increasing interest in their role in the global response to NCDs. ${ }^{910}$ The best recent evidence of CHWs' ability to improve NCD control comes from studies conducted in the USA. A systematic review of randomised controlled trials of CHW-led interventions among Latino patients with diabetes documented decreases in percent haemoglobin A1c of $0.37-0.75$ as well as improved medication adherence when CHWs were added to the standard of care, ${ }^{11}$ and a review among hypertensive patients reported significant improvements in blood pressure in seven of eight randomised controlled trials. ${ }^{12}$ Differences in patient demographics, health infrastructure and CHW roles and responsibilities make it challenging to generalise results from the USA to other settings.

In LMICs, data are limited. There are no studies in Latin America examining the effect of CHW-led interventions on disease outcomes or medication adherence among patients with NCDs. Retrospective studies in rural Uganda ${ }^{13}$ and South Africa ${ }^{14}$ of CHW-led interventions among patients with hypertension have shown an association with improved control, and a national-level retrospective study in Iran documented improved blood pressure and fasting plasma glucose among patients with hypertension and diabetes, respectively. ${ }^{15}$ A CHW-led intervention improved blood pressure in hypertensive patients in least one randomised controlled trial, conducted in Pakistan. ${ }^{16}$ A recent systematic review of CHW-led interventions for NCDs in LMICs found that studies had significant limitations due to study design, high loss to follow-up, failure to report important health outcomes or inappropriate statistical analysis, which limit current understanding of the impact of CHWs in these settings. ${ }^{17}$

There is no national-level CHW programme in Mexico to address NCDs or NCD risk factors, but national strategies emphasise active community participation in addressing the rising burden of NCDs, advocate for the creation of community committees and encourage partnership with non-governmental organisations. ${ }^{45}$ Compañeros en Salud (CES) is a non-governmental organisation working in collaboration with the Mexican Ministry of Health in Chiapas, Mexico, a state with the lowest overall rates of effective health coverage in Mexico. ${ }^{8}$ In this study, we examined whether a CHW-led intervention implemented by CES was associated with changes in disease control and medication adherence among patients with diabetes and/or hypertension. We hypothesised that the intervention would be associated with improvements in these outcomes.

\section{METHODS \\ Study design}

We conducted a prospective cohort study in the context of a programmatic roll-out of a CHW-led intervention. Based on positive findings from a pilot programme in two neighbouring communities, CES made a programmatic decision to scale the intervention to four additional communities in a stepwise fashion. To ensure optimal training, implementation and supervision, they implemented the intervention in one community at a time at 3-month intervals, and to respect equity between communities, they determined the order of the roll-out at random. Because the planned roll-out of the intervention mirrored that of a stepped-wedge trial, ${ }^{18}$ we emulated this study design by collecting outcomes data in all four communities at two occasions at baseline (ie, prior to implementation in any of the four communities) and prior to each expansion to a new community thereafter. We continued data collection until 12 months after the last community received the intervention. Figure 1 shows the timing of data collection and the CHW-led intervention roll-out.

This study was reviewed and approved by institutional review boards of the Brigham and Women's Hospital and the Instituto Tecnológico de Monterrey and conforms to the principles embodied in the Declaration of Helsinki. This study is registered at ClinicalTrials.gov (NCT02549495). Participants provided verbal informed consent which was documented in writing by study staff.

\section{Study population}

We conducted this study in four remote, rural communities with populations of 1500-2500 people in Chiapas, Mexico. Participants were Spanish-speaking. Each community had one clinic. Clinics maintain registries of patients with NCDs that served as the basis for eligibility determination and recruitment. Eligible patients were those who had a diagnosis of diabetes and/or hypertension, resided in a study community, were aged 18 years or older at time of enrolment and were prescribed daily medications by the clinic physician for control of their diabetes and/or hypertension. We excluded patients with secondary hypertension, type 1 diabetes, pregnancy and chronic use of glucocorticoids. We also excluded patients who, after enrolment but prior to the implementation of the intervention in the first community, were removed from diabetes or hypertension treatment by their physician, moved outside the study community, transferred care to another health facility or who no longer carried a diagnosis of diabetes or hypertension. The study size was determined by the number of patients meeting the 


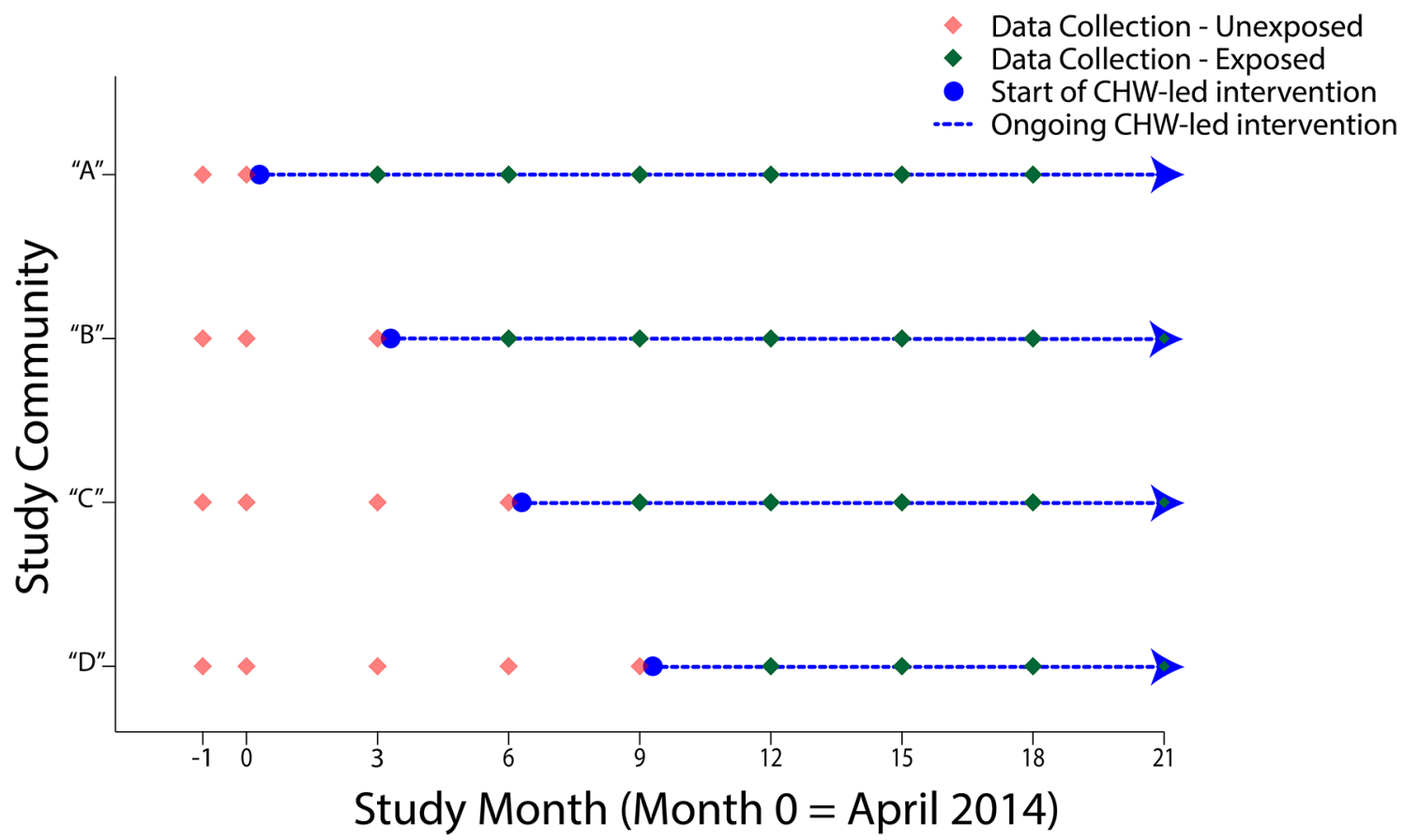

Figure 1 Schematic of stepped-wedge CHW-led intervention roll-out and data collection. CHW, community health workers.

eligibility criteria in the communities where the intervention was to be rolled-out.

\section{NCD care in the study setting}

CES is an affiliate of Partners in Health, a multinational non-governmental organisation with experience with CHW-led interventions to deliver care for a range of medical conditions, including NCDs. ${ }^{19}$ Since February 2012, CES partnered with the Mexican Ministry of Health to rehabilitate, staff and operate existing government primary care clinics. CES provides comprehensive primary care and manages patients with NCDs in accordance with national guidelines. The clinic physician directs care of patients with NCDs, which includes oncemonthly clinic visits, therapy with common oral medications for diabetes (biguanides and sulfonylureas) and hypertension (ACE inhibitors, beta-blockers, diuretics, angiotensin II receptor blockers and calcium channel blockers) and community education. Study patients had Mexico's national health insurance and there are no direct charges for clinic visits or medications. ${ }^{20}$ CES screens all adults in their catchment area for diabetes and hypertension using door-to-door active case finding, works with the Ministry of Health and other suppliers to eliminate medication stock-outs and provides clinical oversight and continuing education to physicians. ${ }^{21}$

\section{Description of CHW activities}

The CHW-led intervention designed by CES, titled 'Acompañantes', follows a 'community-based accompaniment' approach, which has been previously shown to be effective in improving medication adherence and disease outcomes among patients with HIV. ${ }^{22-24}$ In this approach, CHWs serve as a bridge between clinic and patient, promoting medication adherence, reinforcing basic disease education, providing psychosocial support and promoting active case retention. The CHWs in this intervention are women who were nominated at community meetings (either by self or community nomination), wherein CES staff presented the proposal, and then selected on the basis of a formal interview process focusing on leadership potential, motivation as well as basic literacy and education. They were trained in fourtimes-weekly group sessions for 1 month, covering basic pathophysiology, diagnosis and treatment of chronic diseases such as diabetes and hypertension, as well as practical training on the elements of a home visit and the logistical requirements of the role. They also participated in monthly refresher training sessions, covering themes such as brief motivational interviewing, recognising emergencies and complications and navigating interactions with challenging patients. CHWs work longitudinally with four to eight patients, conducting home visits which begin weekly then change in frequency based on a collaborative assessment of the patient's needs by the CHW and clinic physician. CHWs escort patients to clinic visits and meet regularly with clinic physicians to discuss patient management. CHWs are compensated with household food and consumable items, worth a dollar amount approximately equivalent to the monthly stipend given to participants in Prospera (formerly Oportunidades), a Mexican national conditional cash-transfer programme.

\section{Data collection}

Data collection took place from March 2014 to January 2016 (see figure 1). We measured systolic and diastolic blood pressure (blood pressure, in $\mathrm{mm} \mathrm{Hg}$ ) among hypertensive patients, and haemoglobin A1c (A1c, in per 
cent haemoglobin A1c) among patients with diabetes. We measured blood pressure using three measurements using the Omron HEM 7080IT E automated blood pressure cuff. We measured A1c using the Bayer A1c NOW point-of-care device.

We assessed three aspects of self-reported adherence using scales adapted from prior studies of adherence to long-term therapy. ${ }^{2526} 27$ The number of days during the last 5 and 30 days in which medication was not taken was assessed using 5-day recall and 30-day recall, respectively, ${ }^{25}$ and adherence behaviour was assessed using three questions assessed on a Likert scale, similar to the CASE adherence index and adapted to the local context: "How often do you take your medications in the way your doctor instructed?" "How many days a week do you forget to take your pills?" and "Some people choose to stop taking their medications. When was the last time you chose to stop taking your medications?"

Study staff visited patients at home and collected outcomes data in all communities at baseline and at 3-month intervals thereafter, timed just prior to the roll-out of the intervention in a new community. We also administered a questionnaire with basic demographic and income information at the time of enrolment. We classified patients as exposed to the intervention once it had been implemented in their community; however, because data collection occurred just prior to implementation, the first exposed data point for any patient was collected 3 months after implementation in their community. Local clinicians and programme personnel felt the effect of the intervention could be expected within 3 months of implementation.

\section{Outcome definitions}

We defined disease control as A1c $<7$ for patients with diabetes, and blood pressure $<140 / 90$ for hypertensive patients $(<150 / 90$ for patients aged at least 80 years $)$. We classified a patient as optimally adherent by 5 -day recall if $\mathrm{s} /$ he took medications on all of the preceding 5 days, and by 30 -day recall if $\mathrm{s} /$ he took medications on at least 28 of the preceding 30 days. Adherence behaviour was classified as optimal if $\mathrm{s} /$ he reported perfect adherence (five of five possible points on a Likert scale) for two of three questions, and at least near-perfect adherence (four or more of five possible points on a Likert scale) on the third. For patients with diagnoses of both diabetes and hypertension, patients were required to meet the definition of optimally adherent for both sets of medications or controlled disease for both diseases.

\section{Statistical methods and data analysis}

We conducted individual-level mixed effects analyses, which included random intercepts for each individual and community to adjust variances for clustering. We modelled binary outcomes (optimally adherent vs not, controlled vs not) using a logit link. Independent variables included a binary variable to indicate whether the person lived in a community that was exposed to the intervention at each time point and an indicator variable for time point to flexibly model time and minimise residual confounding by time. We conducted stratified analyses to examine whether any effect of the intervention depended on disease (diabetes or hypertension) or baseline disease control (adherence outcomes only) and calculated $\mathrm{P}$ values for differences in ORs using Cochran's Q-test for heterogeneity. Outcome assessments for individuals who withdrew from the study for any reason were included until the time at which they withdrew. We conducted two sensitivity analyses; in the first we adjusted for community, and in the second we excluded 10 individuals who were removed from diabetes and/or hypertension treatment by their providers during the study. Analyses were conducted using SAS V.9.3 (Cary Institute, Cary, North Carolina, USA). Statistical significance was defined at a threshold of $\mathrm{P}<0.05$.

\section{RESULTS}

\section{Overview of enrolment and data completeness}

We screened 142 patients identified through NCD patient registries, and we recruited and enrolled 122 who provided informed consent between 26 February 2014 and 30 April 2014. 14 of these patients (11\%) were excluded prior to intervention implementation (figure 2). Of the 108 patients analysed, 31 (29\%) had a diagnosis of diabetes, $52(48 \%)$ of hypertension and $25(23 \%)$ had both diagnoses. Ninety-one patients (84\%) contributed data through the completion of the study, and 10 of 17 (59\%) of patients withdrawn from the study were withdrawn due to physician discontinuation of therapy (figure 2). Of a total of 902 possible data collection time points corresponding to active study participation (ie, excluding data collection that would have occurred following a withdrawal), we collected data at 866 time points $(96 \%)$.

\section{Description of the study cohort}

We present baseline demographic and outcomes data in table 1 . The majority (66\%) of patients were female, and the median age was 59 years. All three scales recorded a majority of patients reporting suboptimal adherence $(50 \%-58 \%)$ and only $38 \%$ of patients had clinically controlled disease $(17 \%$ and $63 \%$ among those with a diabetes and hypertension diagnosis, respectively).

\section{Clinical control of diabetes and/or hypertension}

Relative to no intervention, receipt of the intervention was associated with a twofold increase in the odds of disease control (time-adjusted OR 2.04, 95\% CI 1.15 to 3.62, $\mathrm{P}=0.02$, $\mathrm{n}=864$ ). Figure 3 shows the prevalence of disease control over time, by intervention status. While disease control rates were higher at the conclusion of the study relative to baseline, we observed greater fluctuations in these rates over time than we did for adherence. In stratified analyses, we did not find evidence that the effect of the intervention on disease control depended on diagnosis (time-adjusted OR $1.78,95 \%$ CI 0.58 to 5.44 for diabetes; time-adjusted OR 


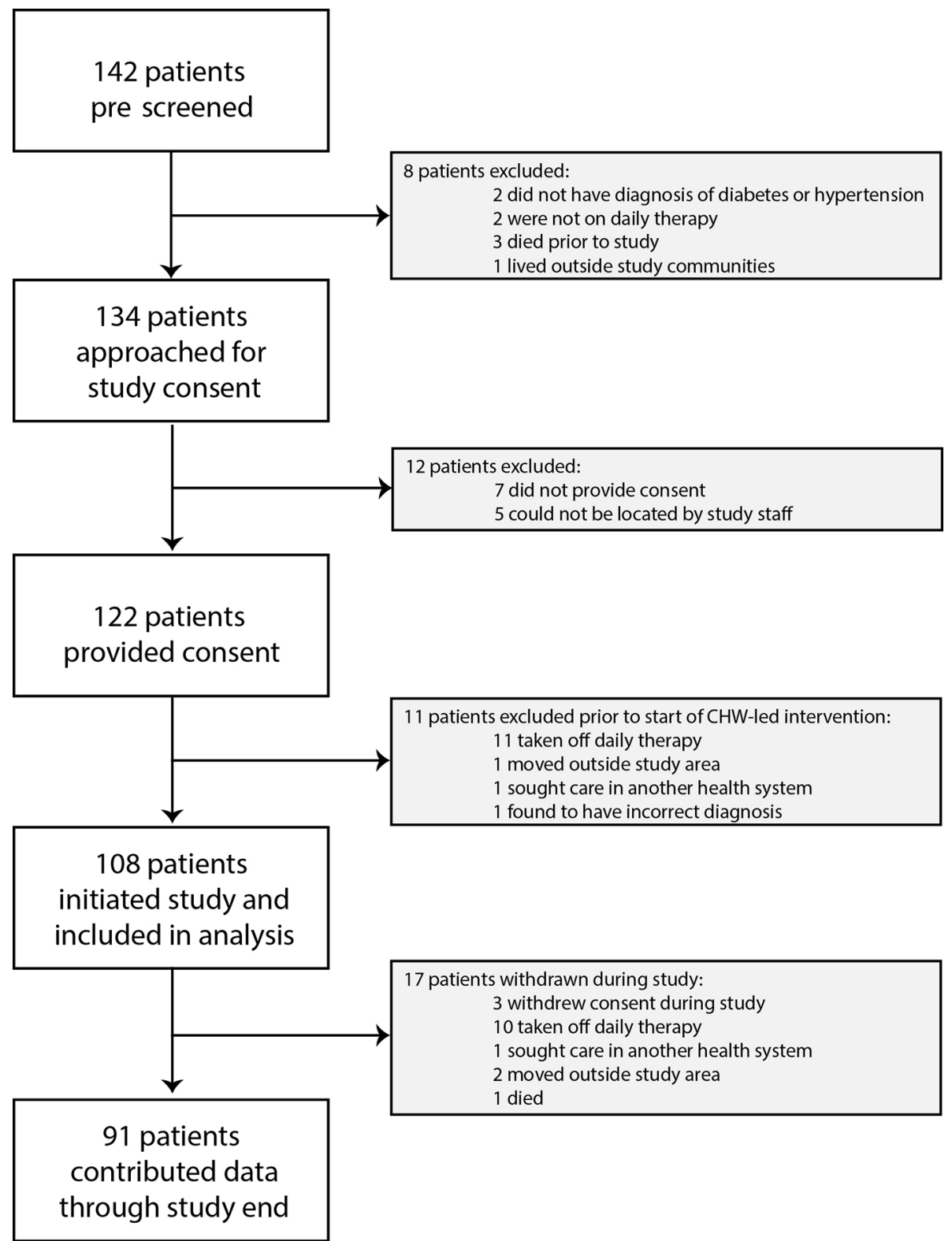

Figure 2 Study enrolment profile. CHW, community health workers.

2.47, $95 \%$ CI 1.54 to 3.96 for hypertension; P value for interaction $=0.59$ ).

\section{Adherence outcomes}

Relative to no intervention, receipt of the intervention was associated with an $86 \%$ increase in the odds of optimal adherence measured by 30-day recall (time-adjusted OR $1.86,95 \%$ CI 1.15 to $3.02, \mathrm{P}=0.01, \mathrm{n}=867$ ), and more than a twofold increase in the odds of positive adherence behaviour (time-adjusted OR 2.29, 95\% CI 1.26 to $4.15, \mathrm{P}=0.007, \mathrm{n}=866$ ). We did not find a statistically significant association between receipt of the intervention and self-reported adherence over the very short term using 5-day recall (time-adjusted OR 1.14, 95\% CI 0.74 to $1.75, \mathrm{P}=0.55, \mathrm{n}=865)$. Figure 4 shows the prevalence of adherence over time, by intervention status, for each of the three adherence scales. Adherence, as measured by all three scales, remained consistently high up to a year following implementation in the last community.
In stratified analyses, we found no evidence that the effect of the intervention on adherence, measured by any of the three scales, depended on disease (results not shown). Similarly, the association between intervention receipt and optimal adherence, as measured by 5-day or 30-day recall, were nearly identical among individuals with and without baseline disease control (results not shown). While the intervention appeared to exert greater positive influence on self-reported adherence behaviour among individuals who lacked baseline control (time-adjusted OR 3.17, 95\% CI 1.31 to $7.72, \mathrm{P}=0.01$ ) as compared with those who had controlled disease at baseline (time-adjusted OR 1.38, $95 \%$ CI 0.79 to $2.41, \mathrm{P}=0.25$ ), this difference was not statistically significant ( $\mathrm{P}$ value for interaction $=0.12$ ).

\section{Sensitivity analysis}

Adjustment for community tended to slightly attenuate effect estimates (5\% for disease control and 30-day recall; 
Table 1 Baseline characteristics of study population, by community

\begin{tabular}{|c|c|c|c|c|c|}
\hline & $\begin{array}{l}\text { Overall } \\
n=108(\%)\end{array}$ & $\begin{array}{l}\text { Community 'A' } \\
n=43(\%)\end{array}$ & $\begin{array}{l}\text { Community 'B' } \\
\mathrm{n}=24(\%)\end{array}$ & $\begin{array}{l}\text { Community 'C' } \\
\mathrm{n}=25(\%)\end{array}$ & $\begin{array}{l}\text { Community ' } D \text { ' } \\
\mathrm{n}=16(\%)\end{array}$ \\
\hline Male & 37 (34) & $19(44)$ & $5(21)$ & 6 (24) & 7 (44) \\
\hline Median age (years) (IQR) ( $n=101)$ & $59(50-72)$ & $59(54-72)$ & $54(48-70)$ & $61(54-73)$ & $55(50-71)$ \\
\hline Has a radio & $66(61)$ & $30(70)$ & $12(50)$ & $17(68)$ & $7(44)$ \\
\hline Has a car or motorcycle & $29(27)$ & $8(19)$ & $6(25)$ & $11(44)$ & $4(25)$ \\
\hline \multicolumn{6}{|l|}{ Type of remuneration for work } \\
\hline Salary & $6(6)$ & $1(2)$ & $0(0)$ & $1(4)$ & $4(25)$ \\
\hline Day labour & $31(29)$ & $15(35)$ & $9(38)$ & $5(20)$ & $2(13$ \\
\hline None & $71(66)$ & $27(63)$ & $15(63)$ & $19(76)$ & $10(63)$ \\
\hline Diabetes diagnosis & $56(52)$ & $29(67)$ & $12(50)$ & $6(24)$ & $9(56)$ \\
\hline Hypertension diagnosis & $77(71)$ & $34(79)$ & $12(50)$ & $23(92)$ & $8(50)$ \\
\hline \multicolumn{6}{|l|}{ Person on whom I rely most for support: } \\
\hline Spouse & $74(69)$ & $29(67)$ & $20(83)$ & $13(52)$ & $12(75)$ \\
\hline Child & $12(11)$ & $4(9)$ & $1(4)$ & $5(20)$ & $2(13)$ \\
\hline Other relative & $18(17)$ & $9(21)$ & $3(13)$ & $4(16)$ & $2(13)$ \\
\hline Neighbour & $4(4)$ & $1(2)$ & $0(0)$ & $3(12)$ & $0(0)$ \\
\hline Controlled diabetes and/or hypertension & $41(38)$ & $18(42)$ & $6(25)$ & $15(60)$ & $2(13)$ \\
\hline Optimally adherent -5 day recall & $45(42)$ & $23(53)$ & 8 (33) & 8 (32) & 6 (38) \\
\hline Optimally adherent-30-day recall & $54(50)$ & $28(65)$ & $11(46)$ & $7(28)$ & $8(50)$ \\
\hline $\begin{array}{l}\text { Optimally adherent - adherence } \\
\text { behaviour }\end{array}$ & $51(47)$ & $25(58)$ & $10(42)$ & $8(32)$ & $8(50)$ \\
\hline
\end{tabular}

$10 \%$ for 5-day recall and adherence behaviour), but did not change overall interpretation or affect statistical significance. Interpretation of findings for primary analyses of adherence and disease control were unchanged in sensitivity analyses excluding the 10 individuals who were removed from treatment by their providers during the study.

\section{DISCUSSION}

We found that a CHW-led intervention was associated with improved disease control and medication adherence among patients with diabetes and/or hypertension

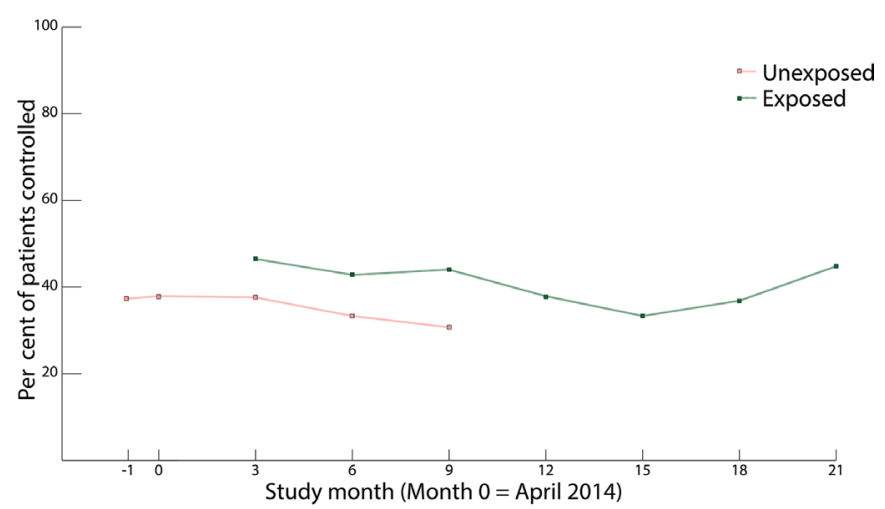

Figure 3 Per cent disease control among the study population over time, by exposure status to community health workers-led intervention. when added to the standard of care in rural Mexico. To the best of our knowledge, this study is among the first prospective studies in any LMIC examining the impact of a CHW-led intervention on disease control and adherence to therapy among patients with NCDs and NCD risk factors, and the first in Latin America. Our results are consistent with evidence from randomised trials in the USA and retrospective observational studies in LMICs among patients with diabetes and/or hypertension. ${ }^{11-15}$

By capitalising on a programmatic stepped roll-out of the CHW-led intervention, we were able to analyse these data as though it were a stepped-wedge trial. This robust approach allowed us to address limitations of the existing literature of CHW-led interventions for NCDs in LMICs. ${ }^{18}$ Because all subjects have periods in which they are unexposed and exposed to the intervention, the study is impervious to confounding by stable individual-level characteristics. Although $16 \%$ of participants were not retained in the study to the end, the majority of patients withdrawn $59 \%$ of patients withdrawn and $9 \%$ of all participants) were withdrawn because their physician discontinued daily therapy, which may actually indicate clinical improvement attributable to the intervention; however, we lacked data to confirm this hypothesis. Sensitivity analyses excluding these individuals were consistent with primary analyses. We followed patients for almost 2 years, providing data on the trend of effect after the immediate postimplementation phase. 


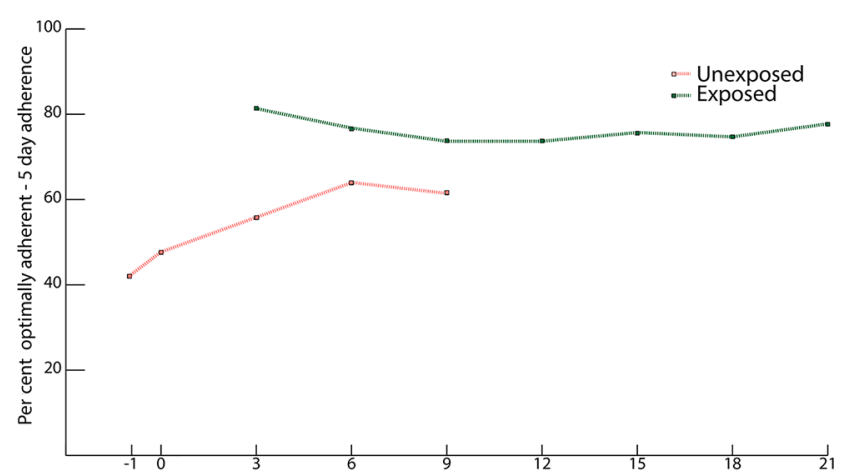

therefore likely generalisable to health systems that aim to do the same.

This study did not attempt to elucidate the mechanism by which CHWs improved clinical control. Possible mechanisms include changes in patients' health-related behaviours (eg, improved self-management due to informational, emotional and instrumental supports provided by CHWs), changes in physician behaviour (eg, improved management due to the CHW's knowledge of, or advocacy for, their patients within a previously binary doctor-patient relationship) and changes in medication adherence. We hypothesise that a significant portion of the improvements we observed can be explained by improved medication adherence. In this study, patterns of improvement in adherence appear similar to those observed for disease control. Suboptimal adherence to therapy impedes NCD control worldwide, and the low baseline rates of adherence observed in this study are in line with WHO estimates of below $50 \%$ adherence to long-term therapy in LMICs. ${ }^{29}$ Difficulty with adherence disproportionately affects patients living in poverty, as many of the barriers prompting poor adherence are related to social vulnerability and lack of agency. CHWs can address many of the common barriers to adherence, including supporting self-management, keeping patients engaged in care and mobilising social supports. In partnership with a health system which eliminates medication stock-outs, personnel shortages and direct user fees, a majority of common barriers to adherence are removed. ${ }^{29} 30$

This study had several limitations. Our relatively small sample size precluded separate disease-specific subgroup analyses of absolute changes in blood pressure and haemoglobin A1c. To maximise statistical power, we included individuals with either or both diseases and created a disease-specific outcome variable to indicate disease control. Future studies will be important in order to gauge the effect size of improvements in blood pressure and haemoglobin A1c due to CHW-led interventions. We observed improved adherence outcomes over time, even prior to intervention implementation. This may be attributed to ongoing programmatic and health system strengthening in all communities over the course of the study, to patients' familiarity with adherence scripts, or to some degree of contamination of the measured effect as CHWs were rolled out to neighbouring communities. A related limitation in the adherence measures was the use of self-report, which is vulnerable to social desirability and recall biases and may result in over-reporting adherence. We observed a discrepancy between high rates of self-reported adherence and much lower rates of disease control, which may be partially explained by overestimation of absolute rates of adherence. Additionally, this discrepancy underscores the fact that although clinical outcomes improved and compare favourably with national-level data in Mexico, ${ }^{6} 7$ they are still lower than the CES programme goal of universal disease control. This 
likely highlights that beyond treatment support, other supporting interventions will be necessary. Such interventions could do more to improve access to care (ie, improving access to insulin) and tackle the social determinants of NCDs (ie, by increasing agency, improving food security and access to healthy foods and increasing exercise); examples may include supporting early child development and education, improving employment opportunities, comprehensive strategies for alcohol and tobacco control and for reducing salt and sugar intake, universal health coverage and other social protection programme. ${ }^{3}$ A reorganisation of the $\mathrm{CHW}$ programme taking this into consideration is currently underway. Nevertheless, any approach that aims to comprehensively address the social determinants of disease and improve outcomes will require new investments in order to build and finance truly functional health systems. Learning how to maximise the highest value inputs while minimising waste will be important for sustaining the political will that is often the main determinant of sustained financing.

We offer evidence from a prospective study documenting an association between a CHW-led intervention and improved clinical control and medication adherence among patients with diabetes and/or hypertension in a rural Latin American setting. Further investigation is warranted to determine which facets of the intervention contribute most greatly to its success, as well as whether the impact differs among different NCDs. Follow-up studies may provide additional important information on the optimal duration of the intervention, the long-term durability of effect and the return on investment of such interventions. Programmes and health systems aiming to improve care of patients with NCDs may consider this study as supportive evidence for the addition of CHW-led interventions to supplement comprehensive primary care systems in rural LMICs.

\section{Author affiliations}

${ }^{1}$ Division of Pediatric Hospital Medicine, Department of Pediatrics, University of California San Francisco School of Medicine, San Francisco, California, USA

${ }^{2}$ Harvard Medical School, Department of Global Health and Social Medicine, Boston, Massachusetts, USA

${ }^{3}$ Harvard T.H. Chan School of Public Health, Boston, Massachusetts, USA

${ }^{4}$ Division of Global Health Equity, Brigham and Women's Hospital, Boston, Massachusetts, USA

${ }^{5}$ Compañeros en Salud, Angel Albino Corzo, Chiapas, Mexico

${ }^{6}$ University of California San Francisco School of Medicine, San Francisco, California, USA

${ }^{7}$ Analysis Group, Inc, Boston, Massachusetts, USA

${ }^{8}$ London School of Hygiene and Tropical Medicine, London, UK

${ }^{9}$ Partners In Health, Boston, Massachusetts, USA

${ }^{10}$ Institute for Health Metrics and Evaluation, University of Washington, Seattle, Washington, USA

Acknowledgements The authors thank the patients for their participation in the study, and the CHWs for their dedicated service to their patients. The authors acknowledge Alejandra Almeida Velasco and all persons involved in the design and implementation of the CHW-led intervention. The authors thank Araceli Gomez Velasco, Edgar Gomez Velasco, Ramiro Cortez Castro and Salomon Benites Santiago for their assistance in data collection. The authors acknowledge and thank Dr Donna Spiegelman for her critical review of the statistical methods. The authors thank the Global Health Research Core at the Department of Global Health and Social Medicine at Harvard University for their support. The authors thank the Harvard Global Health Institute for the graduate fellowship supporting this study. This manuscript is solely the responsibility of the authors and does not represent the official views of the funders.

Contributors DP is the principal investigator, and is responsible for the original conceptualisation, oversight of the study and critical review of the manuscript. PMN led the design of the study, oversaw data collection and drafted the manuscript. PMN, MF, JA, PE, HF, LP, KS and DP contributed to study design. PMN, MF, JA, HF and KS created the data collection tools. AF, KS and HT led data collection teams. PMN, HC, HF and LM oversaw the data collection process and ensured data quality. MF was the lead methodologist and designed the analysis. MF and SG conducted data analysis and produced the figures and tables. All authors participated in the drafting and revision of the manuscript, as well as interpretation of the results. All authors, external and internal, had full access to the study data and take responsibility for the integrity of the data and the accuracy of the data analysis. The lead author affirms the manuscript is an honest, accurate and transparent account of the study being reported; that no important aspects of the study have been omitted and that any discrepancies from the study as planned and registered have been explained.

Funding This study was funded by a grant from the Harvard Global Health Institute. PN's travel costs were supported by the Doris and Howard Hiatt Residency in Global Health Equity and Internal Medicine at the Brigham and Women's Hospital. KS's travel and living costs were supported by the Fulbright US. Student Program. AF's travel and living costs were supported by the Parker Huang Travel Fellowship.

Competing interests All authors have completed the ICMJE uniform disclosure form at www.icmje.org/coi_disclosure.pdf and declare: the research was conducted in collaboration with Partners in Health who independently funded the $\mathrm{CHW}$ intervention, but did not fund the research; otherwise no support from any organisation for the submitted work; DP did short-term consulting work for the Samuel Family Foundation, Eleanor Crook Foundation and Sall Family Foundation.

Patient consent All patient information presented in this manuscript is anonymised.

Ethics approval Partners Human Research Committee (Boston, USA) and Comité de Ética del TEC de Monterrey (Monterrey, México).

Provenance and peer review Not commissioned; externally peer reviewed.

Data sharing statement Anonymised data and technical appendix are available on request from the authors.

Open Access This is an Open Access article distributed in accordance with the Creative Commons Attribution Non Commercial (CC BY-NC 4.0) license, which permits others to distribute, remix, adapt, build upon this work non-commercially, and license their derivative works on different terms, provided the original work is properly cited and the use is non-commercial. See: http://creativecommons.org/ licenses/by-nc/4.0/

(c) Article author(s) (or their employer(s) unless otherwise stated in the text of the article) 2018. All rights reserved. No commercial use is permitted unless otherwise expressly granted.

\section{REFERENCES}

1. UN. Political declaration of the high-level meeting of the general assembly on the prevention and control of non-communicable diseases. http://www.who.int/nmh/events/un_ncd_summit2011/ political_declaration_en.pdf (accessed 19 Apr 2017).

2. WHO. Global status report on noncommunicable diseases. 2010 http://www.who.int/nmh/publications/ncd_report2010/en/ (accessed April 19, 2017)

3. Di Cesare M, Khang YH, Asaria P, et al. Inequalities in noncommunicable diseases and effective responses. Lancet 2013;381:585-97.

4. Secretaría de Salud, México. Estragedia nacional para la prevención y el control del sobrepeso, la obesidad y la diabetes. http:// promocion.salud.gob.mx/dgps/descargas1/estrategia/Estrategia con_portada.pdf (accessed 19 Apr 2017).

5. Secretaría de Salud, México. Programa de acción específico: prevención y control de la obesidad y riesgo cardiovascular 20132018. http://www.cenaprece.salud.gob.mx/descargas/pdf/PAE PrevencionControlObesidadRiesgoCardiovascular2013_2018.pdf (accessed April 19, 2017). 
6. Sosa-Rubí SG, Galárraga O, López-Ridaura R. Diabetes treatment and control: the effect of public health insurance for the poor in Mexico. Bull World Health Organ 2009;87:512-9.

7. Barquera S, Campos-Nonato I, Hernández-Barrera L, et al. Hypertension in Mexican adults: results from the National Health and Nutrition Survey 2006. Salud Publica Mex 2010;52:S63-71.

8. Lozano R, Soliz P, Gakidou E, et al. Benchmarking of performance of Mexican states with effective coverage. Lancet 2006;368:1729-41.

9. Mishra SR, Neupane D, Preen D, et al. Mitigation of noncommunicable diseases in developing countries with community health workers. Global Health 2015;11:43.

10. Perry HB, Zulliger R, Rogers MM. Community health workers in low, middle, and high-income countries: an overview of their history, recent evolution, and current effectiveness. Annu Rev Public Health 2014;35:399-421.

11. Little TV, Wang ML, Castro EM, et al. Community health worker interventions for Latinos with type 2 diabetes: a systematic review of randomized controlled trials. Curr Diab Rep 2014;14:558.

12. Brownstein JN, Chowdhury FM, Norris SL, et al. Effectiveness of community health workers in the care of people with hypertension. Am J Prev Med 2007;32:435-47.

13. O'Neil DS, Lam WC, Nyirangirimana $P$, et al. Evaluation of care access and hypertension control in a community health worker driven non-communicable disease programme in rural Uganda: the chronic disease in the community project. Health Policy Plan 2016;31:878-83.

14. Ndou T, van Zyl G, Hlahane S, et al. A rapid assessment of a community health worker pilot programme to improve the management of hypertension and diabetes in Emfuleni subdistrict of Gauteng Province, South Africa. Glob Health Action 2013;6:19228.

15. Farzadfar F, Murray CJ, Gakidou E, et al. Effectiveness of diabetes and hypertension management by rural primary health-care workers (Behvarz workers) in Iran: a nationally representative observational study. Lancet 2012;379:47-54.

16. Jafar TH, Islam M, Hatcher J, et al. Community based lifestyle intervention for blood pressure reduction in children and young adults in developing country: cluster randomised controlled trial. BMJ 2010;340:c2641.

17. Joshi R, Alim M, Kengne AP, et al. Task shifting for noncommunicable disease management in low and middle income countries--a systematic review. PLoS One 2014;9:e103754.
18. Hussey MA, Hughes JP. Design and analysis of stepped wedge cluster randomized trials. Contemp Clin Trials 2007;28:182-91.

19. Partners in Health. The PIH guide to chronic care integration for endemic non-communicable diseases. http://www.pih.org/library/ the-pih-guide-to-chronic-care-integration-for-endemic-noncommunicable-dise (accessed 19 Apr 2017).

20. Frenk J, González-Pier E, Gómez-Dantés O, et al. Comprehensive reform to improve health system performance in Mexico. Lancet 2006;368:1524-34.

21. Van Wieren A, Palazuelos L, Elliott PF, et al. Service, training, mentorship: first report of an innovative education-support program to revitalize primary care social service in Chiapas, Mexico. Glob Health Action 2014;7:25139.

22. Behforouz HL, Farmer PE, Mukherjee JS. From directly observed therapy to accompagnateurs: enhancing AIDS treatment outcomes in Haiti and in Boston. Clin Infect Dis 2004;38 Suppl 5:S429-36.

23. Thomson DR, Rich ML, Kaigamba F, et al. Community-based accompaniment and psychosocial health outcomes in HIVinfected adults in Rwanda: a prospective study. AIDS Behav 2014;18:368-80.

24. Franke MF, Kaigamba F, Socci AR, et al. Improved retention associated with community-based accompaniment for antiretroviral therapy delivery in rural Rwanda. Clin Infect Dis 2013;56:1319-26.

25. Lu M, Safren SA, Skolnik PR, et al. Optimal recall period and response task for self-reported HIV medication adherence. AIDS Behav 2008;12:86-94.

26. Gonzalez JS, Schneider HE, Wexler DJ, et al. Validity of medication adherence self-reports in adults with type 2 diabetes. Diabetes Care 2013;36:831-7.

27. Mannheimer SB, Mukherjee R, Hirschhorn LR, et al. The CASE adherence index: a novel method for measuring adherence to antiretroviral therapy. AIDS Care 2006;18:853-61.

28. Palazuelos D, Ellis K, Im DD, et al. 5-SPICE: the application of an original framework for community health worker program design, quality improvement and research agenda setting. Glob Health Action 2013;6:19658.

29. WHO. Adherence to long-term therapies: evidence for action. http:// www.who.int/chp/knowledge/publications/adherence_full_report.pdf (accessed 19 Apr 2017)

30. Osterberg L, Blaschke T. Adherence to medication. N Engl J Med 2005;353:487-97. 